\title{
COVID-19 associated viral myocarditis: does it exist?
}

\author{
Vaishali Verma ${ }^{1}$, Sachin Sondhi², Rajesh Sharma ${ }^{1}$, Kunal Mahajan ${ }^{1}$ \\ ${ }^{1}$ Department of Cardiology, Indira Gandhi Government Medical College, Shimla; ${ }^{2}$ ACE Heart and Vascular Institute, \\ Chandigarh, India
}

\section{Dear Editor}

Coronavirus disease 2019 (COVID-19), caused by severe acute respiratory syndrome coronavirus-2 (SARS-CoV-2), is a lethal pandemic that has claimed millions of lives worldwide. While respiratory involvement is the most common and most virulent manifestation of COVID-19, there is enough data to suggest that myocardial injury reflected through elevated troponin levels is seen in around $7-28 \%$ of patients and is related with increased morbidity and mortality (Figure 1) [1,2,3].

Researchers have proposed various mechanisms through which infection with SARS-CoV-2 can lead to myocardial injury [4]. The most commonly proposed hypothesis is the myocardial injury as a result of the cytokine storm induced inflammatory cascade. This inflammatory hypothesis is evident by parallel rise of inflammatory biomarkers like interleukin-1, C-reactive protein, etc., along with the rise in cardiac troponins [4]. Other suggested

Correspondence: Dr. Kunal Mahajan, Department of Cardiology, Indira Gandhi Government Medical College Room number 310, Third Floor, 171001 Shimla, India.

Tel. +91.8628864820.

E-mail: kunalmahajan442@gmail.com

Key words: Viral myocarditis; COVID-19; Coronavirus.

Acknowledgments: None.

Funding: This research did not receive any specific grant from funding agencies in the public, commercial, or not-for-profit sectors.

Conflict of interest: The authors have no conflict of interest to declare.

Contributions: All the authors made substantial contributions to the conception/design of the work, acquisition, analysis, or interpretation of data, drafting the work or revising it critically for important intellectual content. All the authors have read and approved the final version of the manuscript and agreed to be accountable for all aspects of the work.

Received for publication: 15 May 2020.

Accepted for publication: 7 August 2020.

${ }^{\circ}$ Copyright: the Author(s), 2020

Licensee PAGEPress, Italy

Monaldi Archives for Chest Disease 2020; 90:1382

doi: 10.4081/monaldi.2020.1382

This article is distributed under the terms of the Creative Commons Attribution Noncommercial License (by-nc 4.0) which permits any noncommercial use, distribution, and reproduction in any medium, provided the original author(s) and source are credited. mechanisms include acute inflammation-triggered plaque destabilization resulting in acute coronary syndrome, microvascular injury secondary to disseminated intravascular coagulation and thrombosis, and oxygen supply-demand mismatch induced myocardial injury (Type 2 myocardial infarction) [2-4].

Another important mechanism which has been hypothesised is the direct invasion of myocardium by the virus itself resulting in acute viral myocarditis. This hypothesis is based on the observations from previous coronavirus infections including SARS-CoV1 , where viral myocarditis was documented and viral genomes were isolated from the affected myocytes [4,5]. With the ever increasing recognition of occurrence of myocardial injury in COVID-19 and its ill effect on the prognosis, it has become a priority for the researchers to extensively study the pathogenetic mechanisms involved [2,3]. We reviewed the literature and collected all reports pertaining to COVID-19 associated viral myocarditis. Interestingly, we found that the diagnosis of viral myocarditis was made on clinical and imaging grounds in majority of the cases of COVID-19. Some of these reports documented myocardial interstitial inflammation and edema on cardiac magnetic resonance imaging [6-10], while a few studies demonstrated interstitial inflammatory infiltrates on endomyocardial biopsy $[7,11]$. Only in one of the reports, SARS CoV-2 genome could be isolated from endomyocardial biopsy but that too from the interstitial macrophages that had probably infiltrated into the myocardium from the lungs [11]. Similarly one of the autopsy series also didn't reveal any cardiac myocyte necrosis [12]. Thus, all the available data regarding the occurrence of viral myocarditis in COVID-19 is anecdotal and we still do not have any endomyocardial biopsy documentation that SARS-CoV-2 does invade the myocytes. Despite the likely hypothesis that explains the myocardial involvement in COVID disease is an exaggerated inflammatory response caused by the virus, a directed myocardial invasion cannot be excluded because of the lack of data. Endomyocardial biopsy is the definitive diagnostic tool for myocarditis, however, the feasibility of performing endomyocardial biopsies in all suspected cases is questionable especially considering the contagious spread risk, expertise required, and the false negative rate [13]. Furthermore, during this kind of pandemic, performing an endomyocardial biopsy to confirm or exclude acute viral myocarditis in patients with signs and symptoms of acute heart failure, and with suggestive features at cardiac MRI and without other plausible causes is unthinkable. Nevertheless, there are currently no specific antiviral or anti-inflammatory treatment options available against COVID-19 associated myocarditis, therefore, from the therapeutic perspective also, knowing the exact mechanisms underlying the myocardial involvement remains debatable, and would not change the treatment options that include heart failure treatment and arrhythmias control.

On the contrary, endomyocardial biopsy serves as an opportunity for the accurate diagnosis and provides tissues which can be 


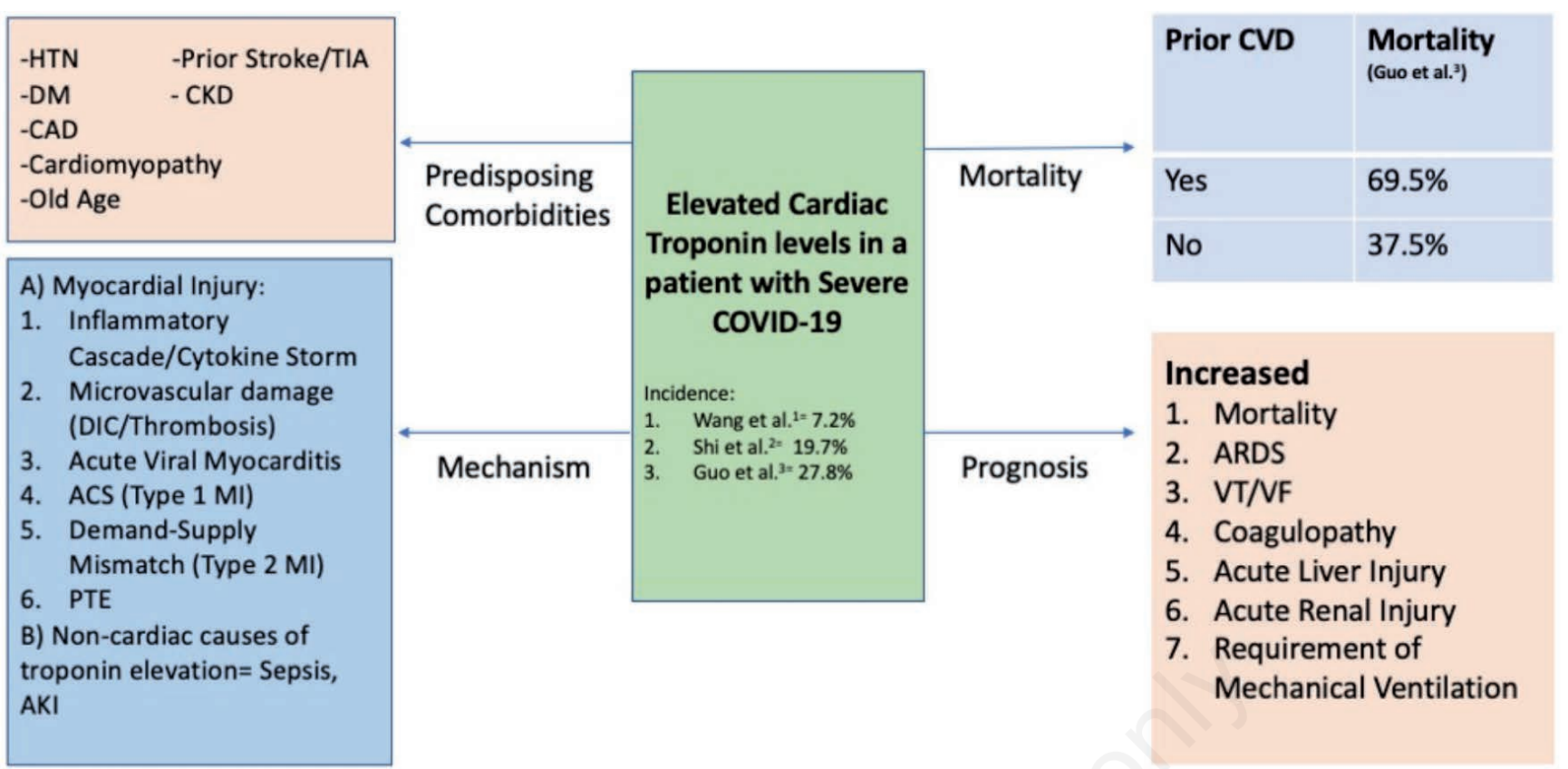

ACS, acute coronary syndrome; AKI, acute kidney injury; ARDS, acute respiratory distress syndrome; CAD, coronary artery disease; CKD, chronic kidney disease; COVID-19, coronavirus disease 2019; CVD, cardiovascular disease; DIC, disseminated intravascular coagulation; DM, diabetes mellitus; HTN, hypertension; MI, myocardial infarction; PTE, pulmonary thromboembolism; TIA, transient ischemic attack; VT/VF, ventricular tachycardia/ventricular fibrillation.

Figure 1. Various mechanisms, comorbidities, and complications associated with acute myocardial injury (defined by elevation in cardiac troponin levels) associated with COVID-19.

explored for the development of specific cardiac biomarkers useful for diagnosing COVID-19 associated myocarditis and may also lead to discovery of new biological pathways for treatment against COVID-19. We suggest that whenever feasible, in case of death of a patient with COVID-19 associated myocarditis, autopsy can be performed to study the gross and microscopic pathology of the heart and to test the presence of viral genomes. Furthermore, if coronary angiography is deemed necessary in a patient with COVID-19 associated cardiac involvement, it is reasonable to simultaneously perform the right heart catheterization and endomyocardial biopsy if the facilities and expertise exist. This may provide new pathogenetic insights and knowledge that is critically needed in this trying time.

\section{References}

1. Wang D, Hu B, Hu C. Clinical characteristics of 138 hospitalized patients with 2019 novel coronavirus-infected pneumonia in Wuhan, China. JAMA 2020;323:1061-9.

2. Shi S, Qin M, Shen B, et al. Association of cardiac injury with mortality in hospitalized patients with COVID-19 in Wuhan, China. JAMA Cardiol 2020;5:802-10.

3. Guo T, Fan Y, Chen M, et al. Cardiovascular implications of fatal outcomes of patients with coronavirus disease 2019 (COVID-19). JAMA Cardiol 2020;5:1-8.

4. Mahajan K, Chandra KS. Cardiovascular comorbidities and complications associated with coronavirus disease 2019. Med J Armed Forces India 2020;76:253-60.
5. Oudit GY, Kassiri Z, Jiang C, et al. SARS-coronavirus modulation of myocardial ACE2 expression and inflammation in patients with SARS. Eur J Clin Invest 2009;39:618-25.

6. Yuan W, Tang X, Zhao X. An 'asymptomatic' driver with COVID-19: atypical suspected myocarditis by SARS-CoV-2. Cardiovasc Diagn Ther 2020;10:242-3.

7. Sala S, Peretto G, Gramegna M, et al. Acute myocarditis presenting as a reverse Tako-Tsubo syndrome in a patient with SARS-CoV-2 respiratory infection. Eur Heart J 2020;41:1 861-2.

8. Kim IC, Kim JY, Kim HA, Han S. COVID-19-related myocarditis in a 21-year-old female patient. Eur Heart J 2020;41:1859.

9. Inciardi RM, Lupi L, Zaccone G, et al. Cardiac involvement in a patient with coronavirus disease 2019 (COVID-19). JAMA Cardiol 2020;5:1-6.

10. Doyen D, Moceri P, Ducreux D, Dellamonica J. Myocarditis in a patient with COVID-19: a cause of raised troponin and ECG changes. Lancet 2020;395:1516.

11. Tavazzi G, Pellegrini C, Maurelli M, et al. Myocardial localization of coronavirus in COVID-19 cardiogenic shock. Eur J Heart Fail 2020;22:911-5.

12. Fox SE, Akmatbekov A, Harbert JL, et al. Pulmonary and cardiac pathology in Covid 19: The first autopsy series from New Orleans. MedRxiv, Preprint posted April 10, 2020. doi: 10.1101/2020.04.06.20050575.

13. Siripanthong B, Nazarian S, Muser D, et al. Recognizing COVID-19-related myocarditis: The possible pathophysiology and proposed guideline for diagnosis and management. Heart Rhythm 2020;17:1463-71. 